\title{
On-line separation of short-lived nuclei by a multi-reflection time-of- flight device
}

\author{
R.N. Wolf ${ }^{\mathrm{a}, *}$, D. Beck ${ }^{\mathrm{b}}$, K. Blaum ${ }^{\mathrm{c}}$, Ch. Böhm ${ }^{\mathrm{c}}$, Ch. Borgmann ${ }^{\mathrm{c}}$, M. Breitenfeldt ${ }^{\mathrm{d}}$, F. Herfurth ${ }^{\mathrm{b}}$, \\ A. Herlert ${ }^{\text {e }}$, M. Kowalska ${ }^{\text {f }}$ S. Kreim ${ }^{\text {c,f }}$, D. Lunney ${ }^{\text {g }}$, S. Naimi ${ }^{\text {g,h }}$, D. Neidherr ${ }^{\text {b }}$, M. Rosenbusch ${ }^{\text {a }}$, \\ L. Schweikhard ${ }^{\text {a }}$, J. Stanja ${ }^{i}$, F. Wienholtz ${ }^{\text {a }}$, K. Zuber $^{\mathrm{i}}$ \\ ${ }^{a}$ Institut für Physik, Ernst-Moritz-Arndt Universität Greifswald, Felix-Hausdorff-Straße 6, 17489 Greifswald, Germany \\ ${ }^{\mathrm{b}}$ GSI Helmholtzzentrum für Schwerionenforschung GmbH, Planckstraße 1, 64291 Darmstadt, Germany \\ c Max-Planck-Institut für Kernphysik, Saupfercheckweg 1, 69117 Heidelberg, Germany \\ d Instituut voor Kern- en Stralingsfysica, KU Leuven, Celestijnenlaan 200d, B-3001 Heverlee, Belgium \\ e FAIR GmbH, Planckstraße 1, 64291 Darmstadt, Germany \\ ${ }^{\mathrm{f}}$ CERN, 1211 Geneva 23, Switzerland \\ ${ }^{g}$ CSNSM-IN2P3-CNRS, Université de Paris Sud 91405 Orsay, France \\ ${ }^{\mathrm{h}}$ RIKEN Nishina Center for Accelerator-based Science, RIKEN, 2-1 Hirosawa, Wako-shi, Saitama 351-0198, Japan \\ i Institut für Kern- und Teilchenphysik, Technische Universität, 01069 Dresden, Germany
}

\section{A R T I C L E I N F O}

\section{Article history:}

Received 5 April 2012

Received in revised form

20 May 2012

Accepted 23 May 2012

Available online 30 May 2012

Keywords:

MR-ToF mass separator

Isobar purification

RIB

ISOLTRAP

Penning trap

Mass spectrometer

\begin{abstract}
A B S T R A C T
A multi-reflection time-of-flight (MR-ToF) mass analyzer has been integrated into ISOLTRAP, the precision mass spectrometer for on-line mass determinations of short-lived nuclides at ISOLDE/CERN. The new instrument improves ISOLTRAP by providing a fast separation of isobaric contaminant species as well as subsequent ion selection using the fast Bradbury-Nielsen gate. Suppression ratios of up to $10^{4}$ and mass-resolving powers of over $10^{5}$ have been reached in off-line experiments. Preliminary data from on-line applications illustrate the benefit and performance of the device and its potential in the context of the ISOLTRAP setup.
\end{abstract}

(c) 2012 Elsevier B.V. All rights reserved.

\section{Introduction}

Penning traps have proven to be the devices of choice with regard to high-precision mass measurements of stable and exotic nuclei $[1,2]$. One of the primary conditions to minimize uncertainties of such a measurement is the availability of pure ion ensembles. In the presence of contaminations, the motion of the ions of interest is altered via the Coulomb interaction [3-6]. In general, the resulting deviations of the trapped-ion frequencies prevent precision experiments. Therefore, it is of utmost importance to ensure contamination-free measurements.

The yield of nuclei produced at on-line facilities decreases far away from the valley of stability whereas usually the ratio of the number of ions of interest and contaminant ions becomes more and more unfavorable. Penning-trap mass spectrometers suffer from

\footnotetext{
* Corresponding author. Tel.: +49383486 4758; fax: +493834864701.

E-mail address: wolf@uni-greifswald.de (R.N. Wolf).
}

systematic effects in presence of contaminants either if the ratio is too large or if the required resolving power is high (usually the case for isobaric or isomeric purification). Thus, one of the most important challenges for ISOLTRAP [7] at ISOLDE/CERN [8], as for other comparable setups installed at on-line facilities [9-14], is the efficient transfer of the ions of interest to the location of the precision measurement and the elimination of all contaminating species. Such contaminations may be delivered from the on-line separator directly or can be created inside the apparatus as products from nuclear decay [15] or charge exchange [16], in particular in the case of stopping in a gas cell [17].

In addition, for nuclides with half-lives on the order of the measurement duration or below ( $\leq 1 \mathrm{~s}$ ), the ion preparation and purification should be as fast as possible to avoid decay losses. Currently, this contamination removal is performed by massselective resonant buffer-gas cooling in a Penning trap [18] which requires several $100 \mathrm{~ms}$. Therefore, a faster mass selection would be a significant improvement for experiments on nuclides with short half-life. For this purpose, a second isobar-purification 
system was developed and installed in 2010 at ISOLTRAP. It is based on a multi-reflection time-of-flight (MR-ToF) technique [19-25] in combination with a fast Bradbury-Nielsen gate (BNG) [26,27] and provides a significantly faster purification without degradation in effectiveness. The system can be used either as

- an auxiliary device for contamination removal in combination with the existing preparation Penning trap,

- an exclusive mass separator for very short-lived nuclides, or

- a fast mass spectrometer in its own right for very short-lived nuclides.

In the following we report on the implementation of the first MR-ToF mass analyzer at a Penning-trap facility for exotic nuclei. In addition to the design of the MR-ToF section and the BNG, the results of off-line as well as of the first on-line experiments on mass separation of short-lived nuclei are presented.

\section{Principle of isobar purification with the MR-ToF/BNG device}

The MR-ToF/BNG isobar-purification principle is illustrated in Fig. 1. The setup consists of an MR-ToF section for the separation of the different ion species with respect to their different massover-charge ratios and thus different arrival times at the BNC where the actual selection of the species of interest takes place.

\subsection{MR-ToF mass separator}

The MR-ToF device uses two electrostatic coaxial ion mirrors to reflect charged-particle bunches. Thus, the mirrors act as an anti-parallel arrangement of two ToF reflectrons and create a multiply-folded flight path [19]. The resulting trajectories can be several hundreds or thousands of meters long all within a compact device of $1 \mathrm{~m}$ length. All ion species $i$ of mass $m_{i}$ and charge state $z_{i}$ start on a well-defined potential $U$ and gain the same average kinetic energy per charge state $E / z_{i}=e U$, where $e$ is the elementary charge. Due to their different velocities $v_{i}=\sqrt{2 U e z_{i} / m_{i}}$ the ions have $m_{i} / z_{i}$-specific revolution times $T_{i} \propto v_{i}^{-1} \propto \sqrt{m_{i} / z_{i}}$. Thus, the difference in ToF between two ion species $\Delta t_{i j} \propto n\left(T_{i}-T_{j}\right)$ increases linearly with the number of revolutions $n$. When the ToF difference becomes larger than the time width $\Delta t$ of the individual ion bunches, $\left|\Delta t_{i j}\right|>\Delta t$, the ions can be ejected from the MR-ToF device towards the BNG.

To achieve the high resolving power $R=m / \Delta m=t /(2 \Delta t)$ necessary to separate, e.g., isobaric species, the total time of flight (ToF) $t$ has to be long compared to the temporal width of an ion bunch $\Delta t$. The total ToF can be extended by increasing the number of revolutions $n$ of the ions in the MR-ToF, since $t \propto n T$. For an increase in resolving power, the signal width has to stay constant. Ideally, the complete ToF system consisting of the flight path from the ion source to the MR-ToF device, the trajectory inside the MRToF device itself, and the flight path from the MR-ToF device to the detector, or to a device for selection, is isochronous with respect to the ion energy. In other words, the flight time should be independent of the different kinetic energies of the ions, originating in particular from different starting positions in the ion source. This can be achieved by applying optimized mirror potentials [28] in combination with the use of a pulsed drift electrode between the ion mirrors that also serves as an injection and ejection device [29]. The upper limit for the time-of-flight separation is given by the maximum time difference between species before they start to approach each other again, $\Delta t_{i j}<T_{i, j} / 2$.

\subsection{Bradbury-Nielsen gate}

The principle of operation of the BNG [26,31] is illustrated in Fig. 2. It consists of two sets of parallel wires in a planar arrangement such that neighboring wires belong to different sets. During the passage period of unwanted species, the gate is activated (closed), i.e. voltages of alternating sign but equal value are applied to neighboring wires, which results in the deflection of the charged particles. When the ions of interest arrive the gate is deactivated (open) until they have passed. A BNG achieves higher time resolutions than parallel-plate deflectors or similar arrangements. The electric field of the activated wires decays exponentially with increasing distance from its plane [31], and thus is negligible at the distance of one wire spacing $d$ in the longitudinal direction, as shown in Fig. 2 (right). The achievable time resolution $\tau_{\text {res }}$, i.e. the ion flight time through the region of the deflecting electric field can be approximated by the ion flight time through twice the wire spacing [31]

$\tau_{\text {res }}=\frac{2 d}{v_{i}}=2 d \sqrt{\frac{m_{i}}{2 z_{i} e U}}$

Thus, for singly charged ions with $m=100 \mathrm{u}$ starting from a potential of $U \approx 3 \mathrm{kV}$ and passing a BNG with $d=0.5 \mathrm{~mm}$, the time resolution is $\tau_{\text {res }} \approx 13 \mathrm{~ns}$. For the selection of ToF-separated ion species, the transition times of the pulsed voltages $\tau_{\text {trans }}$ needed to change the state of the gate from closed to open and vice versa has to be added. Ions that penetrate the field region while the gate potentials are being switched experience a change in kinetic energy which thus broadens the energy distribution of the ensemble. To deflect one species without disturbing the other, the minimum time-of-flight difference between the end of the first ion bunch and the beginning of the second ion bunch at a given relative signal height should therefore not be smaller than $\tau_{\text {res }}+\tau_{\text {trans. }}$ Thus, this condition does not include the bunch width.

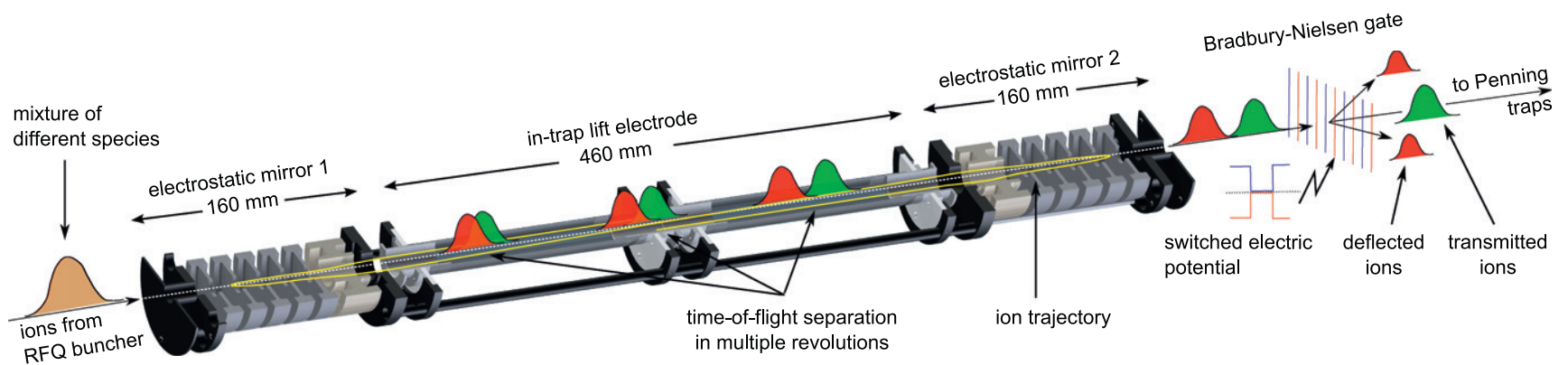

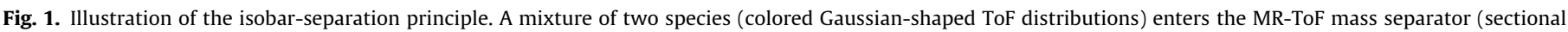

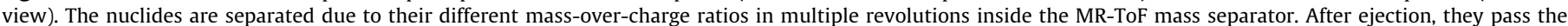
Bradbury-Nielsen gate where the contaminant species are deflected. 

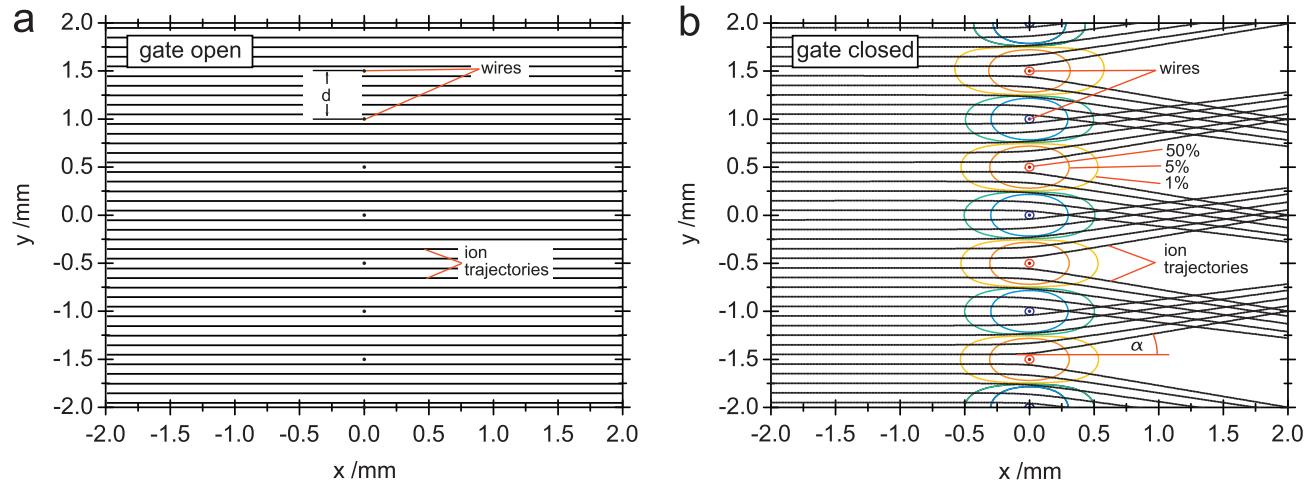

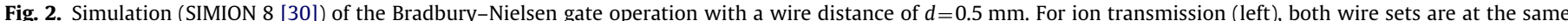
voltage, ideally the potential of the beam line. To deflect the ions (right), the wires are activated. Note the equipotential lines of $50 \%$, $5 \%$ and $1 \%$ of the wire voltage.

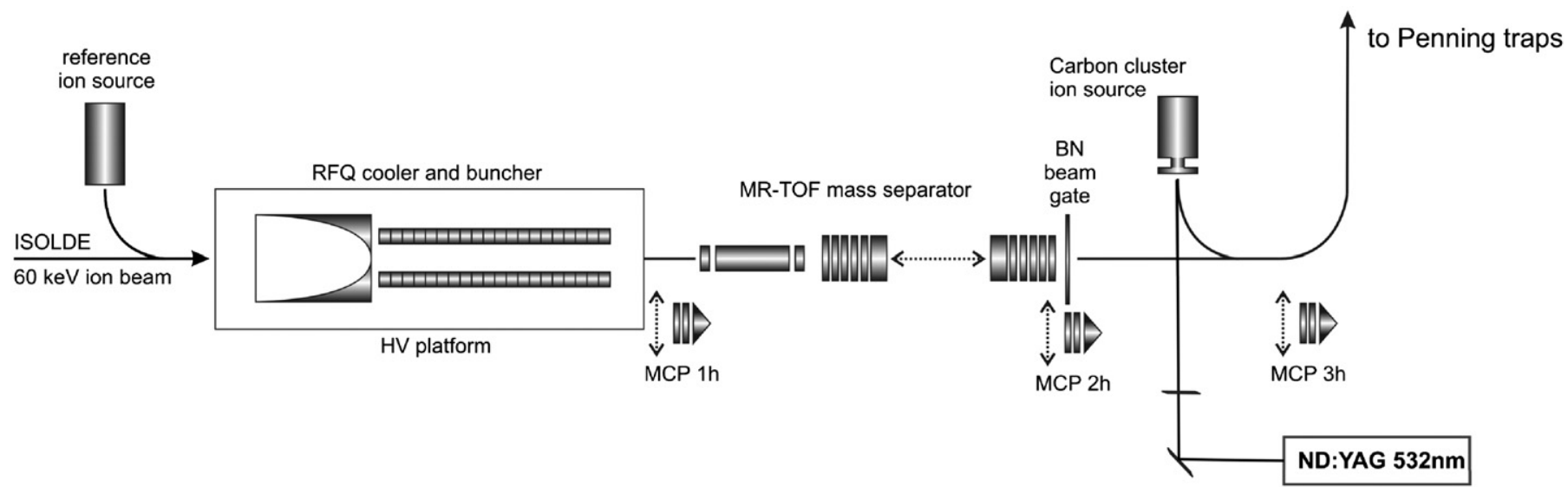

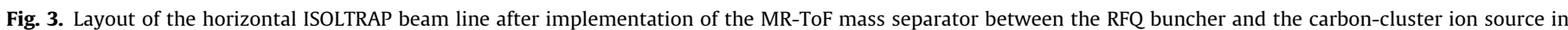
front of the bender that directs the ion bunches towards the vertical Penning traps (not shown). For details see text.

\section{Overview of the ISOLTRAP setup and beam purification in a Penning trap}

The layout of the horizontal ISOLTRAP beam line including the MR-ToF mass separator and the BNG is illustrated in Fig. 3. ISOLTRAP consists of a radiofrequency-quadrupole (RFQ) ionbeam cooler and buncher [32], the new MR-ToF/BNG section, and in the vertical part - not shown in the figure - two Penning traps for the preparation [33] and precision [34] measurement steps mentioned above as well as a tape-station [35] for trapassisted nuclear-decay studies. Two off-line ion sources (alkali ion source and carbon-cluster ion source [36]), one in front of the RFQ buncher and one in front of the preparation Penning trap deliver the stable reference nuclides for the mass measurements. The status of the setup before the implementation of the MR-ToF mass separator has been described in detail in [7]. Only a short review of the parts needed for this work will be given in the following.

The low-energy part of the ISOLDE facility delivers a quasicontinuous ion beam with an ion energy of up to $60 \mathrm{keV}$, in most cases consisting of a mixture of isobaric short-lived and stable nuclides as the separation resolving power of the sector-field magnets is limited to about 7000 without higher-order corrections [8]. For mass measurements, the ions are decelerated electrostatically in front of the ISOLTRAP RFQ buncher located on a high-voltage platform floatable up to $60 \mathrm{kV}$. To accumulate ions and to reduce the beam emittance, the RFQ buncher is filled with high-purity helium buffer gas of $10^{-2}$ mbar. After an accumulation and cooling period, thermalized ion pulses are reaccelerated to $3.1 \mathrm{keV}$ by injection into a switchable drift electrode which transfers the ions to ground potential of the beam line. In the adjacent MR-ToF mass separator, a mass-overcharge separation of the different species can be performed, which are then ejected onto the BNG for selection of the species of interest. After the ions have passed the BNG they are deflected by $90^{\circ}$ to the vertical direction and are injected into the first Penning trap for further purification and cooling as a preparation step for the actual precision mass measurement in the second Penning trap.

The standard method to purify the ion ensemble from contaminating species is the mass-selective resonant buffer-gas centering (so-called "quadrupolar cooling") in a preparation Penning trap: By use of an azimuthal dipolar radiofrequency (RF) excitation the magnetron radius of all ions is simultaneously increased to a value higher than the radius of an aperture used for ejection (in the case of ISOLTRAP, $r=1.5 \mathrm{~mm}$ ) in the endcap electrode of the trap. To re-center only the species of interest mass-selectively, the slow magnetron motion is converted into the fast cyclotron motion by applying an azimuthal quadrupolar $\mathrm{RF}$ excitation at the cyclotron frequency $v_{c}=q B /(2 \pi m)$, with mass $m$, charge $q$, and magnetic field strength $B$. Due to the damping by buffer-gas collisions, the fast cyclotron motion is effectively cooled, leading to a decrease of the cyclotron radius. When the ions are ejected axially, e.g., towards the measurement Penning trap, only the centered ones pass through the aperture, whereas the others collide with this electrode and are lost. Mass-resolving powers of up to 300,000 have been reached with ISOLTRAP's preparation Penning trap [37]. However, such high resolving powers are achieved at very low helium-gas pressures only, where this essential preparation step takes several hundreds of milliseconds. In contrast, if the ensemble is already 
contamination-free and mass separation is not needed, the cooling period can be reduced to only a few milliseconds (at increased helium pressure).

After the preparation, the ions are transferred to the precision Penning trap for the determination of their cyclotron frequency $v_{c}$ in a homogenous magnetic field. To this end, a quadrupolar excitation is performed at several frequencies around the expected cyclotron frequency. In resonance, i.e. at the cyclotron frequency, the magnetron motion is converted to cyclotron motion [38]. This ion-motional response is probed by axial ejection of the ions and a subsequent measurement of their drift time to a micro-channel plate (MCP) detector outside of the strong magnetic field. In the axial magnetic-field gradient the radial (cyclotron) motion is converted to axial kinetic energy, i.e. the conversion becomes visible as a reduction in the time of flight. For short-lived nuclides, a statistical uncertainty in the order of $\delta v_{c} / v_{c} \approx 10^{-9} \ldots 10^{-8}$ can be reached by use of this time-of-flight ion-cyclotron resonance (ToF-ICR) technique [38-40] and the Ramsey excitation scheme [41]. The magnetic-field strength is deduced from the cyclotron frequency measured for a reference ion with a well-known mass.

If necessary, the measurement trap itself can also be used for contaminant reduction, in particular for ions with very close-lying masses: Dipolar RF excitation at the reduced cyclotron frequency can be applied to resolve and remove ions of different isomeric nuclear states $[42,43]$ with a mass-resolving power that is roughly proportional to $v_{c} T_{r f}$ where $T_{r f}$ is the excitation time. As an example, mass-resolving powers of $10^{7}$ have been reached at ISOLTRAP for excitation durations of $T_{r f}=12 \mathrm{~s}$ for $A \approx 85$ at $v_{c} \approx 1 \mathrm{MHz}$. This technique is an important feature also for trapassisted nuclear spectroscopy with the recently installed tapestation setup as it allows beta and gamma spectroscopy of pure isomeric ion ensembles.

\section{Experimental setup}

Fig. 4 shows an overview of the ISOLTRAP MR-ToF purification system which consists of the MR-ToF mass analyzer, injection and ejection ion optics, the BNG and an ion detector. In the following, the technical details of the different parts will be described.

\subsection{Vacuum system}

The MR-ToF device is installed in a CF100/200 cross of $800 \mathrm{~mm}$ length while the adjacent parts are installed in CF100 double-cross chambers. The background pressure determines the mean free path of the ions traveling in the MR-ToF device and thus the transmission on long flight paths. Therefore, an ultrahigh vacuum has to be achieved to avoid ion losses. The injection side is evacuated with a 350-1/s turbo-molecular pump (TMP 1) while at the ejection side a 150-1/s pump (TMP 4) is used. In the MR-ToF central section, a 1500-1/s pump (TMP 2) is used in series with a 50-1/s pump (TMP 3 ) to achieve a low pressure at high gas loads.
Two 250-1/min oil-free scroll pumps maintain a prevacuum of about $1 \times 10^{-3}$ mbar. With closed valves to the neighboring sections, the background pressure of the system is $2 \times 10^{-10} \mathrm{mbar}$ measured at the center of the MR-ToF device, $6 \times 10^{-9}$ mbar at the injection side and $1 \times 10^{-8} \mathrm{mbar}$ at the ejection side. Under normal operation conditions with helium-gas flow from the RFQ buncher $\left(\approx 1 \times 10^{-2} \operatorname{mbar}(\mathrm{He})\right)$ and the preparation Penning trap $\left(\approx 5 \times 10^{-4} \mathrm{mbar}(\mathrm{He})\right)$, the pressure at the central MR-ToF section is $5 \times 10^{-9} \mathrm{mbar}$, and $3 \times 10^{-7} \mathrm{mbar}$ as well as $1 \times 10^{-7} \mathrm{mbar}$ at the injection and ejection crosses, respectively. In order to block the helium gas entering into the MR-ToF section from both sides, two adjustable iris apertures are installed at the injection side and another on the ejection side. A reduction of a factor of six in pressure at the MR-ToF section could be observed by closing the irises to their minimal permissible diameter $(\approx 3 \mathrm{~mm})$ without a decrease in the ion transmission.

\subsection{Injection and ejection ion optics, MCP detector}

To control the radial position and diameter of the injected ion bunches, two sets of $x-y$ steerers and an einzel lens are installed in front of the MR-ToF device (see Fig. 4). Small deflection potentials in the range of a few volts are sufficient to maximize the transmission efficiency of the MR-ToF. These voltages depend on the mass-over-charge ratio of the injected ions. They correct the trajectory variations due to different ejection conditions of the RFQ buncher as well as the mass-dependent transfer due to the residual magnetic stray field of the $4.7 \mathrm{~T}$-magnet of the preparation Penning trap. The einzel lens is used to focus the beam into the activated ion mirror near the reflection point.

In analogy, an einzel lens at the ejection side collimates the ion bunches for further transfer to the MCP detector or to the BNG. The latter devices are both installed on a movable feedthrough (denoted by the arrow in Fig. 4) to allow choosing between either ion monitoring or selection.

\section{3. $M R-T o F$ setup}

The mechanical construction of the MR-ToF device has already been described in detail in [28] and will only be summarized here. It consists of two ion mirrors of six electrodes each, which are separated by a drift section (with length of $460 \mathrm{~mm}$ with an inner diameter of $26 \mathrm{~mm}$ ), which is used as an in-trap lift [29] for ion injection, ejection and time-of-flight focusing. A sketch of the MRToF and a typical potential configuration is shown in Fig. 5. The voltages applied to the mirror electrodes are supplied by highprecision modules (ISEG DPS-series) controlled via a 16-bit digital-to-analog converter (DAC, National Instruments NI6703). All other voltages, for the injection/ejection ion optics and the in-trap lift electrode, are generated by further modules (Applied Kilovolts HP-series) and controlled via the same DAC.

The performance of the MR-ToF separator depends critically on the potentials applied. In particular, a given optimized mirrorpotential distribution has to stay as constant as possible over the whole measurement period. For a discussion on how the different

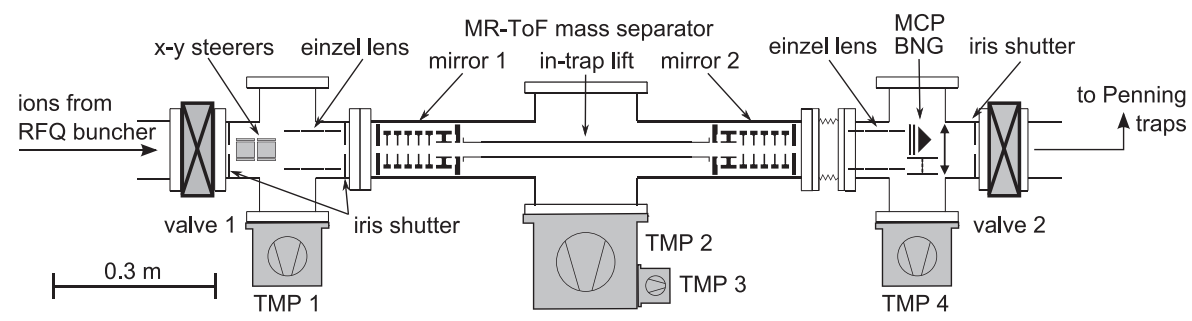

Fig. 4. Time-of-flight isobar-separation section of ISOLTRAP including injection and ejection ion optics and vacuum system. 
spectral components of voltage fluctuations lower the performance, see [29]. The controls of the setup are fully integrated in the experimental control system used at ISOLTRAP (ISOLTRAP CS $[44,45])$. The timing signals for switching the electrode voltages are generated by an FPGA card (National Instruments NI-7811R) programmed as a pulse-pattern generator [46]. This controls among others the activation and deactivation of the in-trap lift electrode via a solid-state MOSFET switch (Behlke HTS 61-03GSM).

\subsection{Bradbury-Nielsen gate}

The BNG construction and weaving mechanism is similar to the one presented in [31]. The gate is constructed by weaving $10 \mu \mathrm{m}$ diameter gold-plated tungsten wire on a frame of polyether ether ketone (PEEK) as shown in Fig. 6. Small groves are milled on the top side of the frame for parallel guiding of the wires at a defined spacing of $d=0.5 \mathrm{~mm}$. On the front side, a comb structure is machined to enhance the distance for the electrically separate contacting. It guides the odd-numbered wires onto the bottom while the even-numbered wires stay on top for some more distance. To fix the wires, strips of PEEK and copper are glued onto the substrate with non-conductive and conductive vacuum-compatible adhesives, respectively. Finally, the wires on the bottom are removed. The gate is placed between two drift

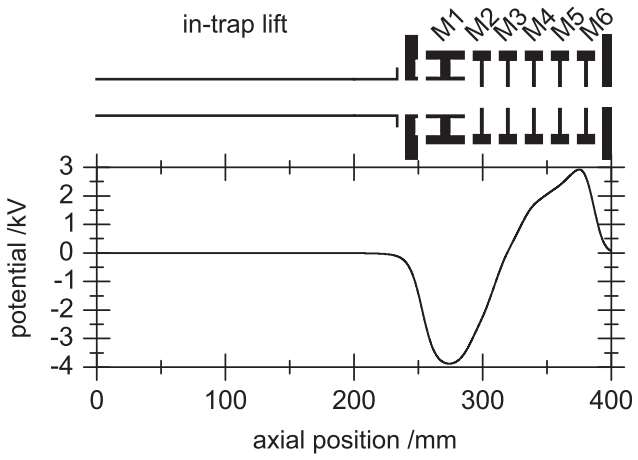

Fig. 5. Axial potential distribution in the MR-ToF device. The first two mirror electrodes (M1 and M2) form a negative electrostatic lens to create stable trajectories. The last four (M3-M6) are on positive voltage to reflect the ions and compensate their energy-dependent ToF. The in-trap lift is on ground potential while the ions are stored and will only be switched for in- or ejection. tube electrodes to prevent ions from hitting non-conductive surfaces, thus charging them. After curing the adhesives and baking the gate at temperatures around $100{ }^{\circ} \mathrm{C}$, no vacuum degradation could be observed. The present gates have an active diameter of $20 \mathrm{~mm}$. Their transmission is about 95\% in the "open" state, which is close to the calculated open area of $98 \%$. The advantage of this method of production is that the PEEK frames for wire spacings down to $0.5 \mathrm{~mm}$ can be produced with standard milling techniques and are therefore easily replaceable.

As described above, the time resolution of a BNG depends on the wire spacing and the voltage transition time on the wires between the states "closed" and "open" and vice versa. Fast pushpull MOSFET switches (model AMX-500-3, CGC Instruments) with voltage transition times of $\tau_{\text {trans }} \approx 20$ ns are used to switch voltages in the range of $U_{B N G}= \pm 250 \mathrm{~V}$.

\section{Off-line operation and performance}

To reach the full operation capability of the MR-ToF/BNG combination, the RFQ buncher had to be modified to provide bunches with a shorter time structure. By decreasing the length of the axial RFQ trapping region from $20 \mathrm{~mm}$ to $10 \mathrm{~mm}$ and by increasing the extraction field strength from $\approx 3 \mathrm{~V} / \mathrm{mm}$ to $\approx 20 \mathrm{~V} / \mathrm{mm}$, the time structure of the bunches measured in front of the MR-ToF (MCP1h, Fig. 3) was reduced from about $1 \mu$ s to $60 \mathrm{~ns}$ (FWHM) at a cooling and bunching efficiency of $10-20 \%$, i.e. the same as reported for the earlier buncher parameters. An initial short time width is necessary to achieve a fast separation in the MR-ToF section. The energy distribution of the ion bunches was determined by use of a repellinggrid arrangement in front of MCP2h (see Fig. 3, with the MR-ToF separator deactivated). The measured energy distribution of about $\Delta E_{90 \%}^{\text {Buncher }} \approx 60 \mathrm{eV}$ is within the energy acceptance of about $100 \mathrm{eV}$ of the preparation Penning trap.

\subsection{MR-ToF performance}

The mean kinetic energy of the ions in the transfer beam line and in the MR-ToF separator is $E_{\text {transfer }}=3.1 \mathrm{keV}$ and $E=2.1 \mathrm{keV}$, respectively, where the reduction is due to the in-trap lift method [29]. Mass-resolving powers of up to $R_{F W H M}=m / \Delta m \approx 2 \times 10^{5}$ at storage times of $t \approx 30 \mathrm{~ms}$ (about 1000 revolutions for ${ }^{133} \mathrm{Cs}$ or 2000 revolutions for ${ }^{39} \mathrm{~K}$ ions, respectively) have been achieved. As an example, Fig. 7 shows the corresponding time-of-flight

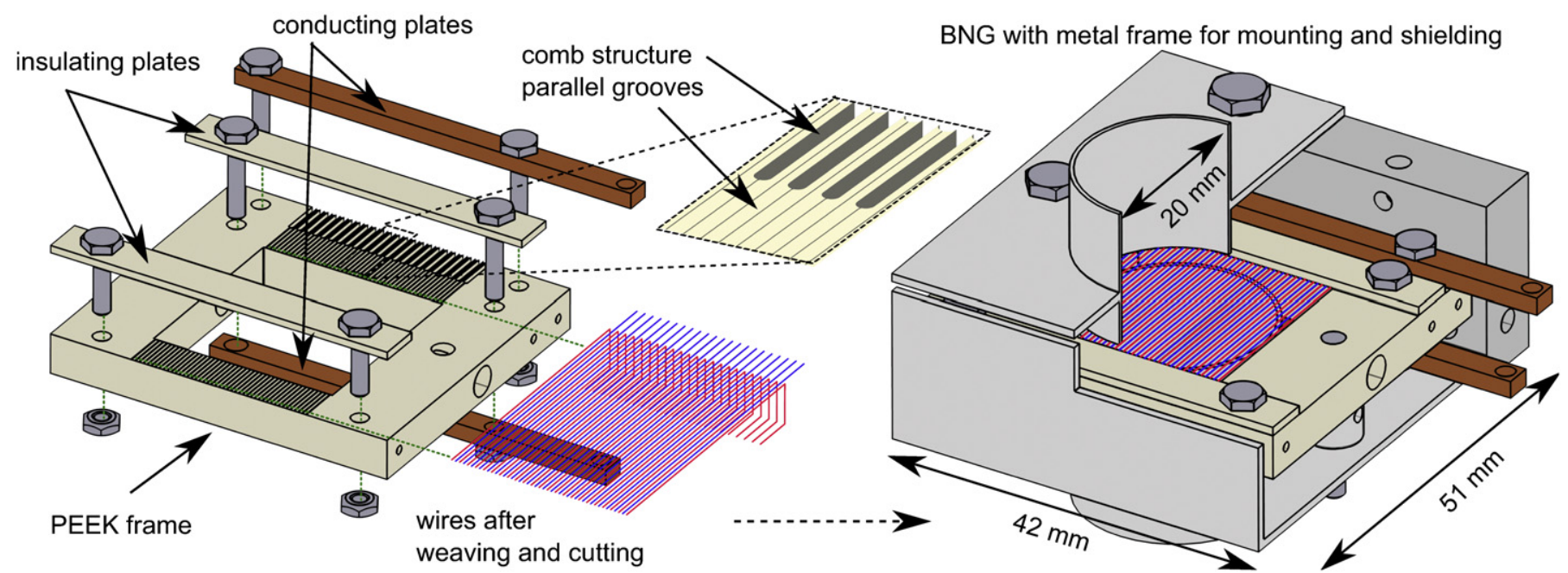

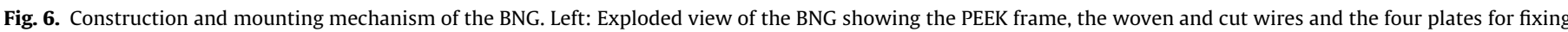

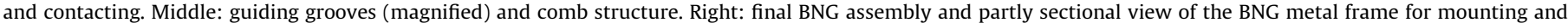
shielding of the insulating parts. 
spectrum for ${ }^{39} \mathrm{~K}$. It was acquired with a multi-channel analyzer adding 200 single-shot spectra of MCP2h. The repetition rate was approximately $1.5 \mathrm{~Hz}$. Thus, the recording time of $\approx 120 \mathrm{~s}$ in this example is short compared to the typical duration of mass measurements with the precision Penning trap which can take several hours if very low statistical uncertainties are to be obtained. Therefore, long-term voltage drifts on the MR-ToF electrodes have to be considered as well (see next paragraph). Fig. 8 (top) shows the mass-resolving power as a function of the time of flight for ${ }^{133}$ Cs ions. Eq. (8) from [29]

$R=\frac{m}{\Delta m}=\frac{t}{2 \Delta t}=\frac{t_{s}+n T_{0}}{2 \sqrt{\Delta t_{t h}^{2}+\left(\Delta t_{s}-n T_{0}\left(\partial \delta_{T} / \partial \delta_{E}\right) \Delta \delta_{E}\right)^{2}}}$

was used to fit the mass-resolving power for the fixed experimental parameters, i.e. the revolution time $T_{0} \approx 27.7 \mu$ s and the ToF difference between the ion source's time-focus plane and the detector, $t_{s} \approx 30 \mu \mathrm{s}$. The resulting free fit parameters are: the turn-around time $\Delta t_{t h} \approx 58 \mathrm{~ns}$ in the ejection section of the RFQ buncher when ejecting (which is the lower limit of the time width), the additional time-spread due to MR-ToF-external flight time $\Delta t_{\mathrm{s}} \approx 105 \mathrm{~ns}$, and the ToF-dispersion relation with respect to energy $\left(\partial \delta_{T} / \partial \delta_{E}\right) \Delta \delta_{E} \approx 5 \times 10^{-6}$ (see [29] for further details).

The dependence of the average time of flight on the mirror voltages is shown in Fig. 9. Variations of mirror electrode 5 (M5) have the largest influence, namely $(\delta t / t) /\left(\Delta V /\left.V\right|_{M 5}\right)=0.5$ because it is closest to the reflection point and therefore defines the penetration depth. For example, a fluctuation of $\Delta V /\left.V\right|_{M 5}=5 \times$ $10^{-6}$ in the spectrum shown in Fig. 7 would result in a ToF center shift of $\delta t / t=2.5 \times 10^{-6}$, i.e. $\delta t \approx 75 \mathrm{~ns}$ for a flight time of $t \approx 30 \mathrm{~ms}$. Thus, the signal width would be doubled and hence the mass-resolving power reduced by a factor of 2 . As mentioned above the Penning-trap mass measurements can last several hours, and thus the possible shift of the time of flight of the ions of interest in the MR-ToF has to be taken into account. The major contribution to the long-term instability is given by the temperature variation in the laboratory of $\approx 1 \mathrm{~K}$ between day and night and a temperature coefficient of the voltage sources of $\Delta V / V=5 \times 10^{-5} / \mathrm{K}$

The transmission of the MR-ToF is mostly affected by the injection focusing and steering of the pulses delivered by the RFQ buncher. Small changes in the focus position and angle with respect to the optical axis can increase the losses in the first few

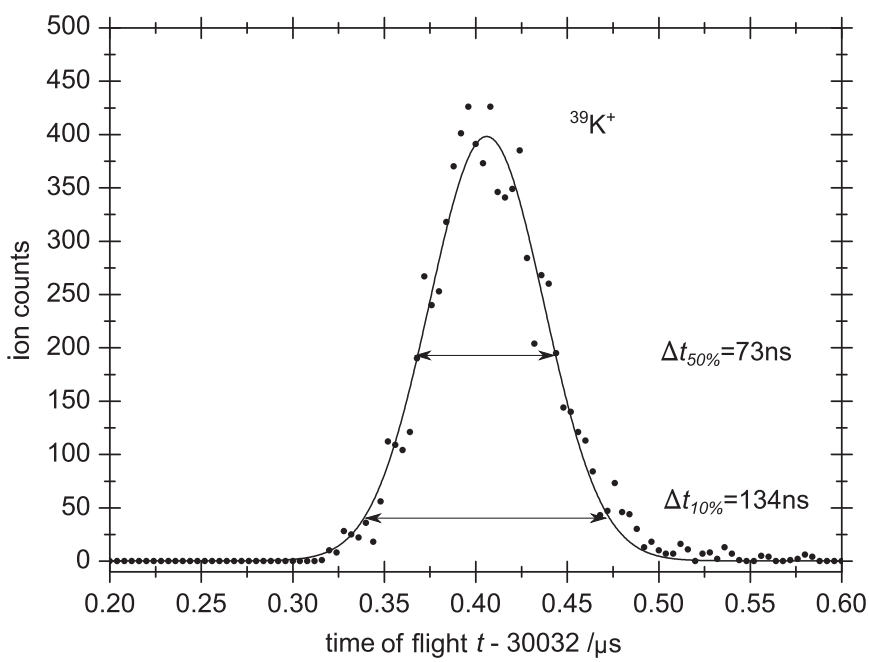

Fig. 7. Ion counts (dots) and Gaussian fit (line) of ${ }^{39} \mathrm{~K}$ ions from the reference ion source at a total flight time of about $t \approx 30 \mathrm{~ms}$ (corresponding to 2000 revolutions at mass $A=39$ in the MR-ToF separator). The mass-resolving power is $R_{F W H M} \approx 2 \times 10^{5}$ and $R_{10 \%} \approx 1 \times 10^{5}$.

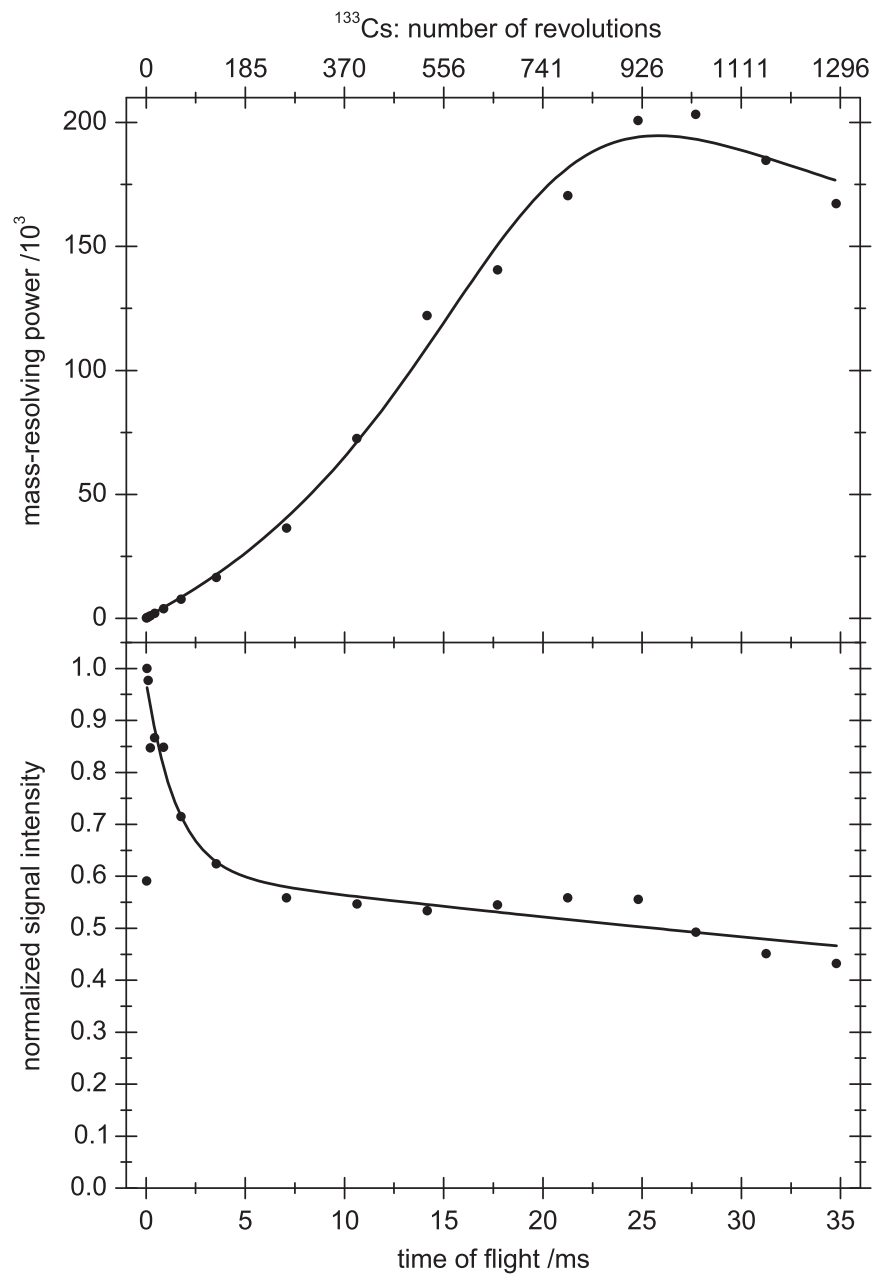

Fig. 8. Mass-resolving power (top) and signal intensity (bottom) of ${ }^{133} \mathrm{Cs}$ with respect to the time of flight (number of revolutions) in the MR-ToF mass separator. The solid lines are fits to the data points. See text for details.

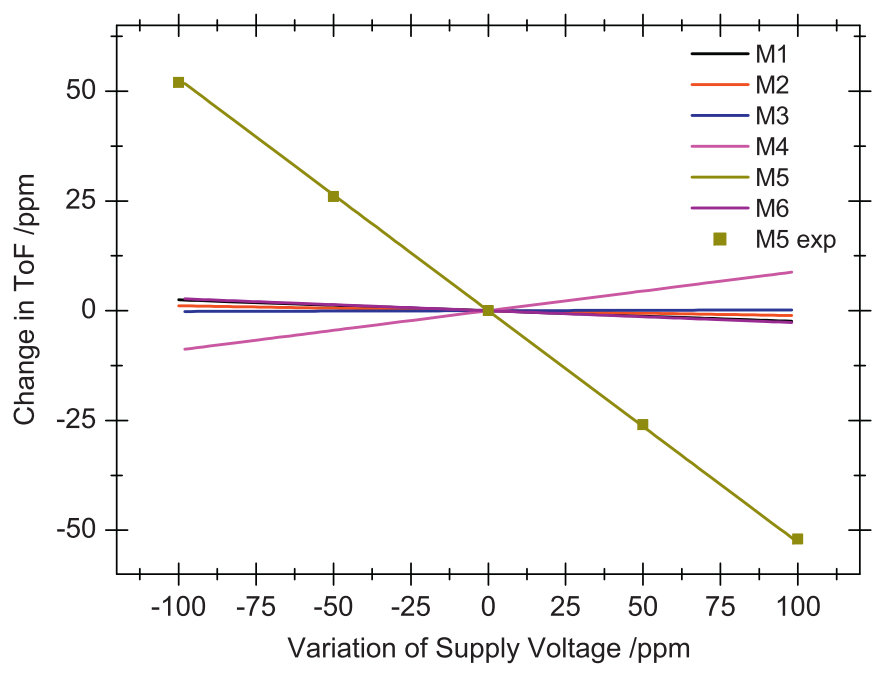

Fig. 9. Time-of-flight deviation from the nominal value as a function of the mirror-electrode voltages (mirror electrodes $1-6=\mathrm{M} 1-\mathrm{M} 6$ ) for a given kinetic ion energy. For the electrode numbering, see Fig. 5. The simulations agree with measured data points (of ${ }^{133} \mathrm{Cs}$ ) for the electrode M5

milliseconds of trapping as shown in Fig. 8 (bottom). The solid line shows an exponential fit to the data with two constants, $\tau_{1} \approx 1.5 \mathrm{~ms}$ and $\tau_{2} \approx 130 \mathrm{~ms}$. The fast losses are most likely a 
result of a mismatch of the pulse emittance and the capture acceptance of the MR-ToF separator. Furthermore, residual stray magnetic fields in the order of $1 \mathrm{mT}$ due to the superconducting magnet of the preparation Penning trap and due to the steel girders of the surrounding support structures of ISOLTRAP influence the injection and trapping efficiency mass dependently. A transmission of about $50 \%$ can be maintained even for storage times up to a few tens of milliseconds. Losses due to collisions with residual gas have a minor influence on the transmission due to the pressure of $<10^{-8}$ mbar, resulting in the high time constant $\tau_{2}$.

The overall efficiency of the ISOLTRAP setup for low ion yields is on the order of $0.5-2 \%$, deduced from the ratio of the beam current in the ISOLDE beam line in front of the RFQ buncher and the ion events counted on the MCP detector used for the mass measurement. The transmission loss in the MR-TOF mass separator of $50 \%$ is thus small compared to other section of ISOLTRAP.

\subsection{BNG performance}

The suppression factor of ions as a function of the wire-voltage difference of the BNG is shown in Fig. $10 .{ }^{85} \mathrm{Rb}$ ions from the alkali reference ion source were ejected towards the BNG while it was either "open" or "closed". The transmitted ions were captured in the preparation Penning trap and ejected towards an MCP detector after the cooling procedure as explained above. Below $\Delta U_{B N G}=440 \mathrm{~V}$, corresponding to $U_{B N G}= \pm 220 \mathrm{~V}$ on the wire sets, the suppression is low since the deflection angle is too small to

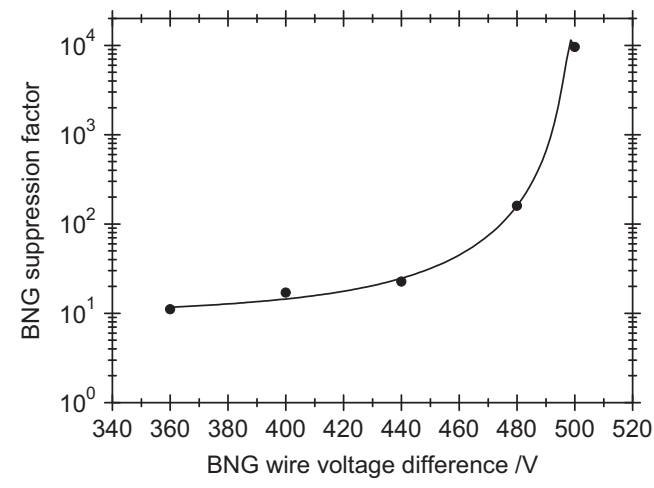

Fig. 10. BNG suppression (ratio of ${ }^{85} \mathrm{Rb}$ ions transmitted for BNG open vs. BNG closed) as a function of the wire voltage difference, solid line to guide the eye. For the determination of the deflection efficiency the dark-count rate has been subtracted. prevent the ions from being transported by the subsequent ion optics into the preparation Penning trap. Above deflection voltages of $\Delta U_{B N G}=480 \mathrm{~V}$ the ratio between ions that passed the "closed" and the "open" gate decreases by orders of magnitudes, i.e. the deflection angle is sufficient. A reduction of four orders of magnitude has been achieved at $\Delta U_{B N G}=500 \mathrm{~V}$, which was the maximum voltage used to avoid damaging the BNG. However, this is no general limit for the suppression ratio.

\section{On-line application}

In the following, the potential of the new sections at ISOLTRAP is illustrated by first on-line data. Fig. 11 (left) shows a time-offlight spectrum of short-lived $A=80$ zinc and rubidium ions (with half-lives of $540 \mathrm{~ms}$ and $33.4 \mathrm{~s}$, respectively).

After the ions had been transferred through the ISOLDE HRS sector-field mass separator and captured at the ISOLTRAP RFQ buncher they performed 400 revolutions in the MR-ToF, corresponding to a flight time of $t \approx 8.6 \mathrm{~ms}$. Thus, the ion species were separated by $\Delta t_{80 \mathrm{Zn}, 80 \mathrm{Rb}} \approx 1.2 \mu$ s while their bunch width was $\Delta t \approx 70 \mathrm{~ns}$. This corresponds to a mass-resolving power of over 60,000 , fully sufficient to separate both species by multiple signal widths. After the separation the ${ }^{80} \mathrm{Zn}$ ions were selectively transmitted through the BNG to the preparation Penning trap. There, they were cooled for only $15 \mathrm{~ms}$ at a high helium buffergas pressure, i.e. a second mass-separation step was not necessary, before they were forwarded to the precision Penning trap. Thus, the distribution of isobar separation and ion-sample preparation into two separate steps and on two devices reduced the overall processing time by a factor of ten to $<25 \mathrm{~ms}$. Fig. 11 (right) shows a ToF-ICR spectrum of ${ }^{80} \mathrm{Zn}$ ions including the detailed ToF ion distributions within the individual time-of-flight spectra [47]. Obviously, there is no ${ }^{80} \mathrm{Rb}$ contamination, as these ions would show up at flight times of about $400 \mu$ s at the center of the ${ }^{80} \mathrm{Zn}$ resonance. This proves that the MR-ToF and BNG system were used effectively to purify the ensemble.

The fast ion separation in the MR-ToF device will allow highprecision mass measurements of nuclides with half-lives as low as a few milliseconds. The feasibility of this approach is demonstrated in Fig. 12 which shows an MR-ToF time-of-flight spectrum of the $A=137$ nuclides barium, praseodymium, neodymium, promethium and samarium for a MR-ToF storage time of $\approx 22.5 \mathrm{~ms}$. Such spectra allow the determination of the mass of one ion species from the known masses of the others, which act as internal mass references. This implies the advantage that they are exposed to the same fluctuations and drifts of the system, which
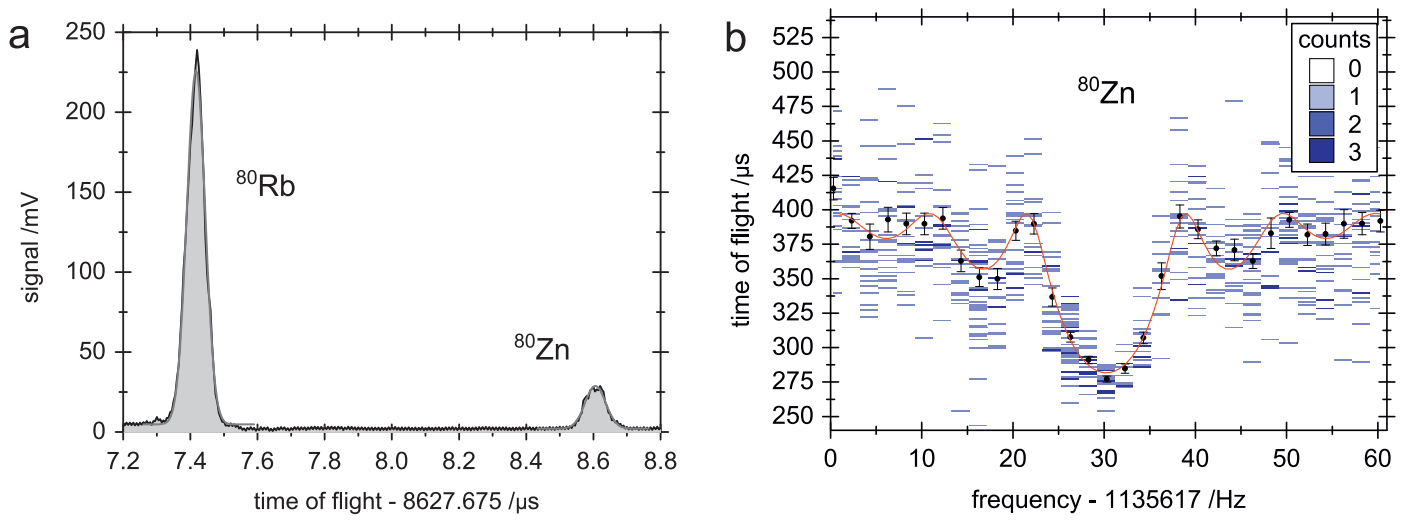

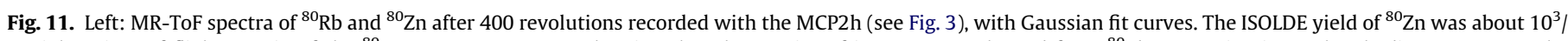

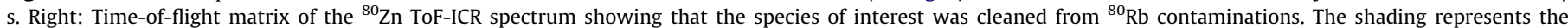
number of counts recorded in the respective time-of-flight bin. 


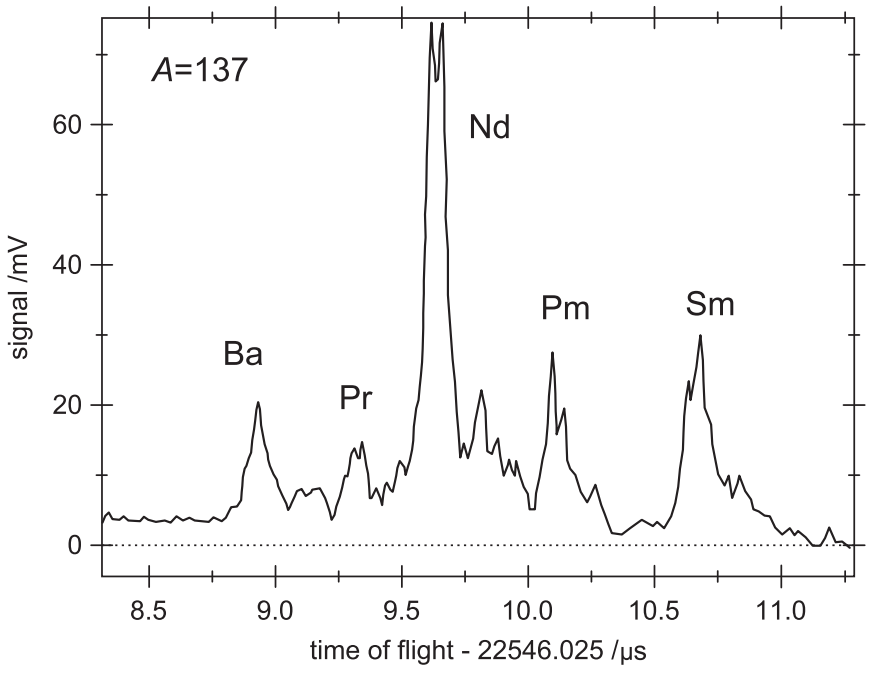

Fig. 12. MR-ToF time-of-flight spectrum of exotic nuclides at mass number $A=137$ after 800 revolutions in the MR-ToF.

reduces the systematic uncertainty significantly. This proof-ofprinciple data has been taken in a region of the nuclides which has already been explored by Penning-trap measurements [48]. In these previous experiments, the necessary mass resolving power of 50,000 was reached by excitation durations of several $100 \mathrm{~ms}$ while the MR-ToF mass separator storage time for comparable mass-resolving power was just $\approx 22.5 \mathrm{~ms}$. The relative deviation to the literature value of e.g. ${ }^{137} \mathrm{Nd}$, by using ${ }^{137} \mathrm{Ba}$ and ${ }^{137} \mathrm{Sm}$ as calibrants, derived from this data is $\Delta m / m \approx 1 \times 10^{-6}$ which is in good agreement taking the 1-sigma width of the ToF signals $\left(>1 \times 10^{-6}\right)$. A more detailed investigation of the uncertainties will be performed in the upcoming measurement periods.

\section{Summary and outlook}

The implementation of an MR-ToF mass separator in the online Penning-trap mass spectrometer ISOLTRAP has been described in detail and its performance has been characterized with respect to systematic measurements during both off-line and on-line experiments. The results confirm that the MR-ToF mass separator is a valuable addition to the ISOLTRAP setup. While it adds further complexity as it is the fourth ion-storage device besides the RFQ buncher and two Penning traps, it has significantly improved the purification speed and facilitates measurements of nuclides with shorter half-lives, of capital importance for advancing nuclear physics. These data are under evaluation and will be published soon.

To evaluate the effect of ion-ion interaction [49-51] on the performance of the MR-ToF device, numerical and experimental investigations are under way.

\section{Acknowledgments}

This work was supported by the German Federal Ministry for Education and Research (BMBF) (06GF9102 and 06GF9101I) and the European Union seventh framework through ENSAR (contract no. 262010).

\section{References}

[1] K. Blaum, Physics Reports 425 (2006) 1

[2] L. Schweikhard, G. Bollen, International Journal of Mass Spectrometry 251 (2-3) (2006) 85.
[3] G. Bollen, H.-J. Kluge, M. König, T. Otto, G. Savard, H. Stolzenberg, R.B. Moore, G. Rouleau, G. Audi, Phys. Rev. C 46 (1992) R2140.

[4] S. Sturm, K. Blaum, M. Breitenfeldt, P. Delahaye, A. Herlert, L. Schweikhard, F. Wenander, in: Non-neutral Plasma Physics VII: Workshop on Non-Neutral Plasmas 2008, vol. 1114, 2009, p. 185.

[5] A. Herlert, Ch. Borgmann, D. Fink, Ch. Holm Christensen, M. Kowalska, S. Naimi, Hyperfine Interactions 199 (2011) 211.

[6] A. Gustafsson, A. Herlert, F. Wenander, Nuclear Instruments and Methods in Physics Research, Section A 626 (2011) 8.

[7] M. Mukherjee, D. Beck, K. Blaum, G. Bollen, J. Dilling, S. George, F. Herfurth, A. Herlert, A. Kellerbauer, H.-J. Kluge, S. Schwarz, L. Schweikhard, C. Yazidjian, European Physical Journal A 35 (2008) 1.

[8] E. Kugler, Hyperfine Interactions 129 (2000) 23.

[9] V. Kolhinen, S. Kopecky, T. Eronen, U. Hager, J. Hakala, J. Huikari, A. Jokinen, A. Nieminen, S. Rinta-Antila, J. Szerypo, J. Äystö, Nuclear Instruments \& Methods in Physics Research, Section A 528 (2004) 776.

[10] G. Savard, J. Wang, K. Sharma, H. Sharma, J. Clark, C. Boudreau, F. Buchinger, J. Crawford, J. Greene, S. Gulick, A. Hecht, J. Lee, A. Levand, N. Scielzo, W. Trimble, J. Vaz, B. Zabransky, International Journal of Mass Spectrometry 251 (2006) 252

[11] J. Dilling, R. Baartman, P. Bricault, M. Brodeur, L. Blomeley, F. Buchinger, J. Crawford, J.R.C. López-Urrutia, P. Delheij, M. Froese, G.P. Gwinner, Z. Ke, J.K.P. Lee, R.B. Moore, V. Ryjkov, G. Sikler, M. Smith, J. Ullrich, J. Vaz, T. Collaboration, International Journal of Mass Spectrometry 251 (2006) 198

[12] M. Block, D. Ackermann, K. Blaum, A. Chaudhuri, Z. Di, S. Eliseev, R. Ferrer, D. Habs, F. Herfurth, F.P. Heßberger, S. Hofmann, H.-J. Kluge, G. Maero, A. Martín, G. Marx, M. Mazzocco, M. Mukherjee, J.B. Neumayr, W.R. Plaß, W. Quint, S. Rahaman, C. Rauth, D. Rodríguez, C. Scheidenberger, L. Schweikhard, P.G. Thirolf, G. Vorobjev, C. Weber, The European Physical Journal D_Atomic, Molecular, Optical and Plasma Physics 45 (2007) 39.

[13] J. Ketelaer, J. Krämer, D. Beck, K. Blaum, M. Block, K. Eberhardt, G. Eitel, R. Ferrer, C. Geppert, S. George, F. Herfurth, J. Ketter, S. Nagy, D. Neidherr, R. Neugart, W. Nörtershäuser, J. Repp, C. Smorra, N. Trautmann, C. Weber, Nuclear Instruments \& Methods in Physics Research, Section A 594 (2008) 162.

[14] R. Ringle, G. Bollen, A. Prinke, J. Savory, P. Schury, S. Schwarz, T. Sun, Nuclear Instruments \& Methods in Physics Research, Section A 604 (2009) 536

[15] A. Herlert, D. Beck, K. Blaum, F. Carrel, P. Delahaye, S. George, C. Guénaut, F. Herfurth, A. Kellerbauer, H.-J. Kluge, D. Lunney, M. Mukherjee, L. Schweikhard, C. Yazidjian, New Journal of Physics 7 (2005) 44.

[16] A. Herlert, S. Baruah, K. Blaum, P. Delahaye, M. Dworschak, S. George, C. Guénaut, U. Hager, F. Herfurth, A. Kellerbauer, M. Marie-Jeanne, S. Schwarz, L. Schweikhard, C. Yazidjian, International Journal of Mass Spectrometry 251 (2006) 131.

[17] P. Schury, G. Bollen, M. Block, D. Morrissey, R. Ringle, A. Prinke, J. Savory, S. Schwarz, T. Sun, Hyperfine Interactions 173 (2006) 165.

[18] G. Savard, S. Becker, G. Bollen, H.-J. Kluge, R. Moore, T. Otto, L. Schweikhard, H. Stolzenberg, U. Wiess, Physics Letters A 158 (1991) 247.

[19] H. Wollnik, M. Przewloka, International Journal of Mass Spectrometry and Ion Processes 96 (1990) 267

[20] Y. Ishida, M. Wada, Y. Matsuo, I. Tanihata, A. Casares, H. Wollnik, Nuclear Instruments \& Methods in Physics Research, Section B 219-220 (2004) 468.

[21] A. Verentchikov, M. Yavor, Y. Hasin, M. Gavrik, Technical Physics 50 (2005) 73.

[22] A. Verentchikov, M. Yavor, Y. Hasin, M. Gavrik, Technical Physics 50 (2005) 82.

[23] W.R. Plaß, T. Dickel, M. Petrick, D. Boutin, Z. Di, T. Fleckenstein, H. Geissel, C. Jesch, C. Scheidenberger, Z. Wang, European Physical Journal Special Topics 150 (2007) 367.

[24] A. Piechaczek, V. Shchepunov, H. Carter, J. Batchelder, E. Zganjar, S. Liddick, H. Wollnik, Y. Hu, B. Griffith, Nuclear Instruments \& Methods in Physics Research, Section B 266 (2008) 4510

[25] P. Schury, K. Okada, S. Shchepunov, T. Sonoda, A. Takamine, M. Wada, H. Wollnik, Y. Yamazaki, European Physical Journal A 42 (2009) 343.

[26] N. Bradbury, R. Nielsen, Physical Review 49 (1936) 388.

[27] W.R. Plaß, T. Dickel, U. Czok, H. Geissel, M. Petrick, K. Reinheimer, C. Scheidenberger, M.I Yavor, Nuclear Instruments \& Methods in Physics Research, Section B 266 (2008) 4560.

[28] R.N. Wolf, M. Eritt, G. Marx, L. Schweikhard, Hyperfine Interactions 199 (2011) 115.

[29] R.N. Wolf, G. Marx, M. Rosenbusch, L. Schweikhard, International Journal of Mass Spectrometry 313 (2012) 8

[30] SIMION 8, Scientific Instrument Services, Inc.

[31] O.K. Yoon, I.A. Zuleta, M.D. Robbins, G.K. Barbula, R.N. Zare, Journal of the American Society for Mass Spectrometry 18 (2007) 1901.

[32] F. Herfurth, J. Dilling, A. Kellerbauer, G. Bollen, S. Henry, H.-J. Kluge, E. Lamour, D. Lunney, R. Moore, C. Scheidenberger, S. Schwarz, G. Sikler, J. Szerypo, Nuclear Instruments \& Methods in Physics Research, Section A 469 (2001) 254.

[33] H. Raimbault-Hartmann, D. Beck, G. Bollen, M. König, H.-J. Kluge, E. Schark, J. Stein, S. Schwarz, J. Szerypo, Nuclear Instruments \& Methods in Physics Research, Section B 126 (1997) 378.

[34] G. Bollen, S. Becker, H.-J. Kluge, M. König, R. Moore, T. Otto, H. RaimbaultHartmann, G. Savard, L. Schweikhard, H. Stolzenberg, Nuclear Instruments and Methods in Physics Research Section A: Accelerators, Spectrometers, Detectors and Associated Equipment 368 (1996) 675

[35] M. Kowalska, S. Naimi, J. Agramunt, A. Algora, G. Audi, D. Beck, B. Blank, K. Blaum, Ch. Böhm, M. Breitenfeldt, E. Estevez, L. Fraile, S. George, F. Herfurth, A. Herlert, A. Kellerbauer, D. Lunney, E. Minaya-Ramirez, 
D. Neidherr, B. Olaizola, K. Riisager, M. Rosenbusch, B. Rubio, S. Schwarz, L. Schweikhard, U. Warring, European Physical Journal A 42 (2009) 351.

[36] K. Blaum, G. Bollen, F. Herfurth, A. Kellerbauer, H.-J. Kluge, M. Kuckein, E. Sauvan, C. Scheidenberger, L. Schweikhard, European Physical Journal A 15 (2002) 245.

[37] M. Rosenbusch, Ch. Böhm, Ch. Borgmann, M. Breitenfeldt, A. Herlert, M. Kowalska, S. Kreim, G. Marx, S. Naimi, D. Neidherr, R. Schneider, L. Schweikhard, International Journal of Mass Spectrometry 314 (2012) 6.

[38] G. Bollen, R.B. Moore, G. Savard, H. Stolzenberg, Journal of Applied Physics 68 (1990) 4355.

[39] M. König, G. Bollen, H.-J. Kluge, T. Otto, J. Szerypo, International Journal of Mass Spectrometry and Ion Processes 142 (1995) 95

[40] G. Gräff, H. Kalinowsky, J. Traut, Zeitschrift für Physik A Hadrons and Nuclei 297 (1980) 35.

[41] S. George, G. Audi, B. Blank, K. Blaum, M. Breitenfeldt, U. Hager, F. Herfurth, A. Herlert, A. Kellerbauer, H.-J. Kluge, M. Kretzschmar, D. Lunney, R. Savreux S. Schwarz, L. Schweikhard, C. Yazidjian, Europhysics Letters 82 (2008) 50005.

[42] J. Van Roosbroeck, C. Guénaut, G. Audi, D. Beck, K. Blaum, G. Bollen, J. Cederkall, P. Delahaye, A. De Maesschalck, H. De Witte, D. Fedorov, V.N. Fedoseyev, S. Franchoo, H.O.U. Fynbo, M. Górska, F. Herfurth, K. Heyde, M. Huyse, A. Kellerbauer, H.-J. Kluge, U. Köster, K. Kruglov, D. Lunney, V.I. Mishin, W.F. Mueller, S. Nagy, S. Schwarz, L. Schweikhard, N.A. Smirnova, K. Van de Vel, P. Van Duppen, A. Van Dyck, W.B. Walters, L. Weissman, C. Yazidjian, Physical Review Letters 92 (2004) 112501.
[43] K. Blaum, D. Beck, G. Bollen, P. Delahaye, C. Guénaut, F. Herfurth A. Kellerbauer, H.-J. Kluge, D. Lunney, S. Schwarz, L. Schweikhard, C. Yazidjian, Europhysics Letters 67 (2004) 586.

[44] CS-Framework project page, 〈https://sourceforge.net/projects/cs-frame work $/>$.

[45] D. Beck, K. Blaum, H. Brand, F. Herfurth, S. Schwarz, Nuclear Instruments \& Methods in Physics Research, Section A 527 (2004) 567.

[46] F. Ziegler, D. Beck, H. Brand, H. Hahn, G. Marx, L. Schweikhard, Nuclear Instruments \& Methods in Physics Research, Section A 679 (2012) 1-6.

[47] T. Eronen, V.-V. Elomaa, U. Hager, J. Hakala, J.C. Hardy, A. Jokinen, A. Kankainen, I.D. Moore, H. Penttila, S. Rahaman, S. Rinta-Antila, J. Rissanen, A. Saastamoinen, T. Sonoda, C. Weber, J. Aysto, Physical Review Letters 100 (2008) 132502.

[48] D. Beck, F. Ames, G. Audi, G. Bollen, F. Herfurth, H.-J. Kluge, A. Kohl, M. König, D. Lunney, I. Martel, R. Moore, H. Raimbault-Hartmann, E. Schark, S. Schwarz, M. de Saint Simon, J. Szerypo, The European Physical Journal A-Hadrons and Nuclei 8 (2000) 307.

[49] H.B. Pedersen, D. Strasser, S. Ring, O. Heber, M.L. Rappaport, Y. Rudich, I. Sagi, D. Zajfman, Physical Review Letters 8705 (2001) 055001.

[50] P. Bolotskikh, D. Grinfeld, A. Makarov, M. Monastyrskiy, Nuclear Instruments \& Methods in Physics Research, Section A 645 (2011) 146.

[51] M.W. Froese, M. Lange, S. Menk, M. Grieser, O. Heber, F. Laux, R. Repnow, T. Sieber, Y. Toker, R. von Hahn, A. Wolf, K. Blaum, The decay of ion bunches in self-bunching mode, New Journal of Physics, accepted for publication. 